Supporting Information for Publication

\title{
Understanding the Transformation of 2D Layered Perovskites to 3D Perovskites in the Sonochemical Synthesis
}

Modasser Hossain, ${ }^{a}$ Trupthi Devaiah Chonamada, ${ }^{a, b}$ and Pralay K. Santra*a ${ }^{a}$ Centre for Nano and Soft Matter Sciences (CeNS), Shivanapura, Bengaluru, India562162.

bManipal Academy of Higher Education (MAHE), Manipal, India-576104

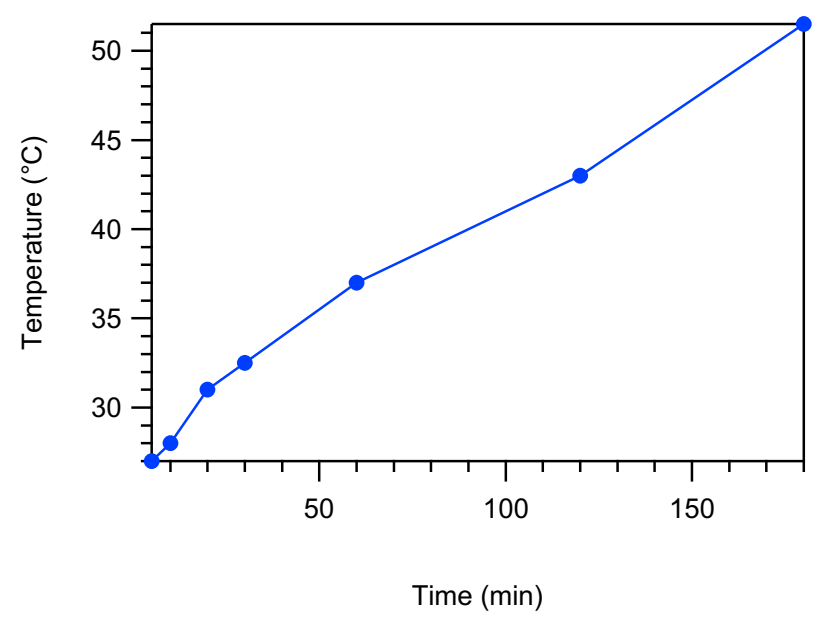

Figure S1: Temperature variation due to the sonication over a period of time. 
(a)

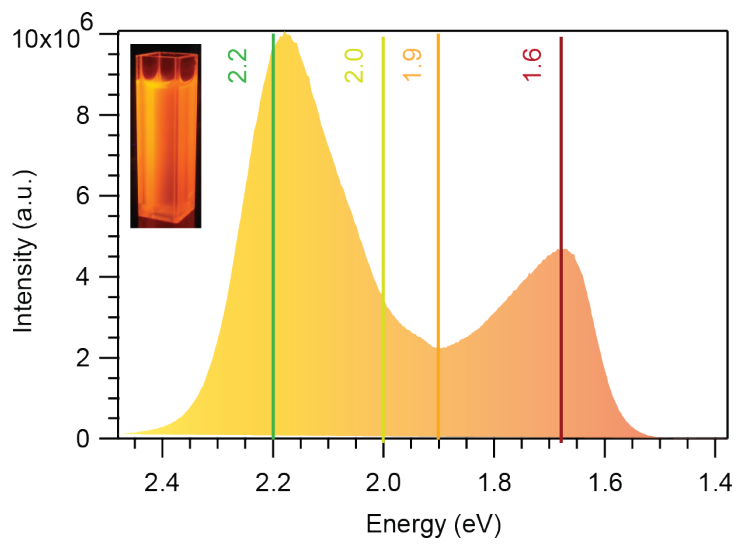

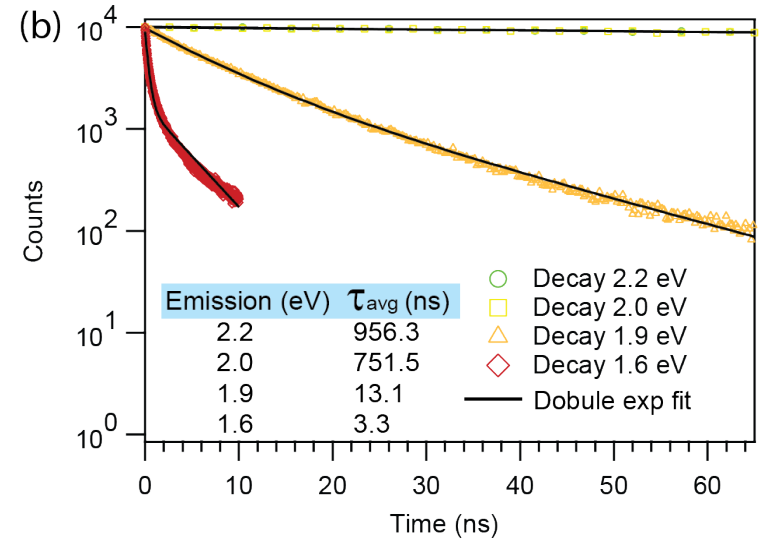

Figure S2: (a) PL spectra of the reaction mixtures synthesized at $30^{\circ} \mathrm{C}$ for 30 minutes of reaction. The inset shows the digital photographs of the reaction mixture under UV illumination. (b) Time correlated single photon counting (TCSPC) fluorescence decay curves of the same sample. Average lifetimes, calculated by fitting with double exponential decay function, are shown in the inset. The average lifetime decreases systematically with an increase in the layer numbers $(n)$ of the 2D layered perovskites to 3D MAPI.

(a)

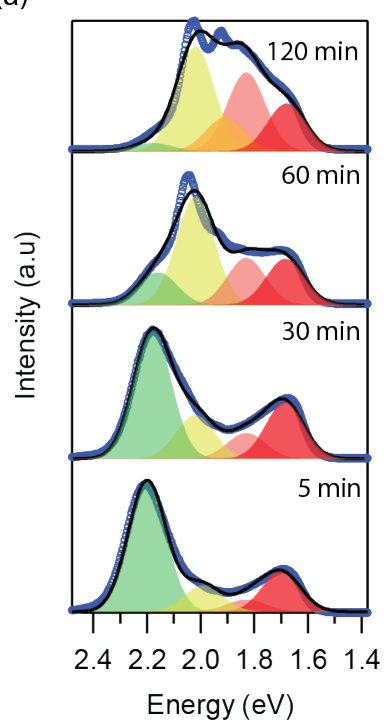

(b)

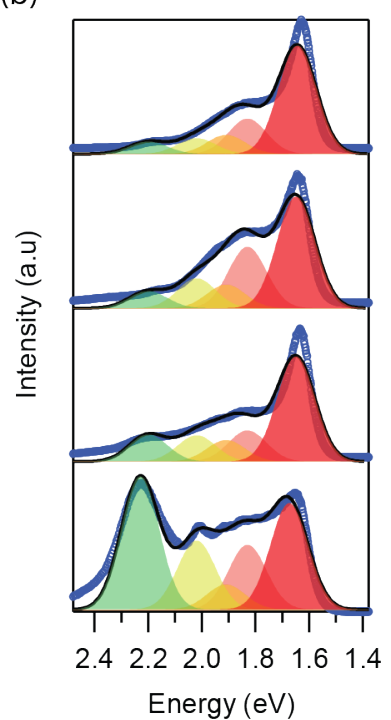

(c)

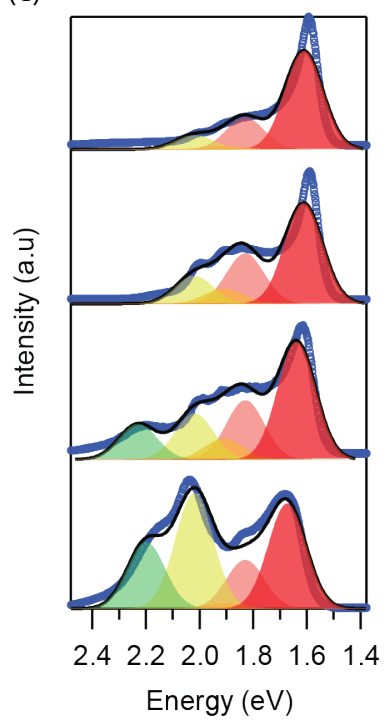

Figure S3: Deconvoluted PL spectra of the reaction mixtures collected at sonication time of 5 minutes, 30 minutes, 60 minutes and 120 minutes, syntheses at (a) $30^{\circ} \mathrm{C}$, (b) $50 \mathrm{C}^{\circ}$ and (c) $80^{\circ} \mathrm{C}$. The blue circled trace represents the experimental PL spectra, the black trace represents theoverall fit. In the deconvoluted spectra, the green, yellow, orange, light red and red colors (shaded region) correspond to the PL emission peak at $2.2 \mathrm{eV}, 2.0 \mathrm{eV}, 1.9 \mathrm{eV}, 1.8 \mathrm{eV}$ and $1.6 \mathrm{eV}$ respectively. The fitting was done using Gaussian functions in Igor pro. 
Table S1: The PL maximum for a different number of $2 \mathrm{D}$ layers of $\mathrm{MAPb}_{3}$ structure. ${ }^{1}$

\begin{tabular}{cc}
\hline Layer number $(\mathbf{n})$ & Wavelength $(\mathbf{n m})$ \\
\hline 1 & 514 \\
2 & 572 \\
3 & 608 \\
4 & 645 \\
5 & 675 \\
$\infty$ & 763 \\
\hline
\end{tabular}

Table S2: PL emission peak position for $n=\infty$ of 3D MAPI calculated from the fitted PL spectra (Figure S3).

\begin{tabular}{ccccc}
\hline Temperature & \multicolumn{4}{c}{ PL emission at $\mathbf{n}=\mathbf{\infty}$} \\
\hline & $\mathbf{5}$ mins & $\mathbf{3 0}$ mins & $\mathbf{6 0}$ mins & $\mathbf{1 2 0}$ mins \\
\hline 30 & $1.70 \mathrm{eV}$ & $1.68 \mathrm{eV}$ & $1.68 \mathrm{eV}$ & $1.68 \mathrm{eV}$ \\
50 & $1.67 \mathrm{eV}$ & $1.65 \mathrm{eV}$ & $1.65 \mathrm{eV}$ & $1.64 \mathrm{eV}$ \\
80 & $1.67 \mathrm{eV}$ & $1.64 \mathrm{eV}$ & $1.61 \mathrm{eV}$ & $1.61 \mathrm{eV}$ \\
\hline
\end{tabular}



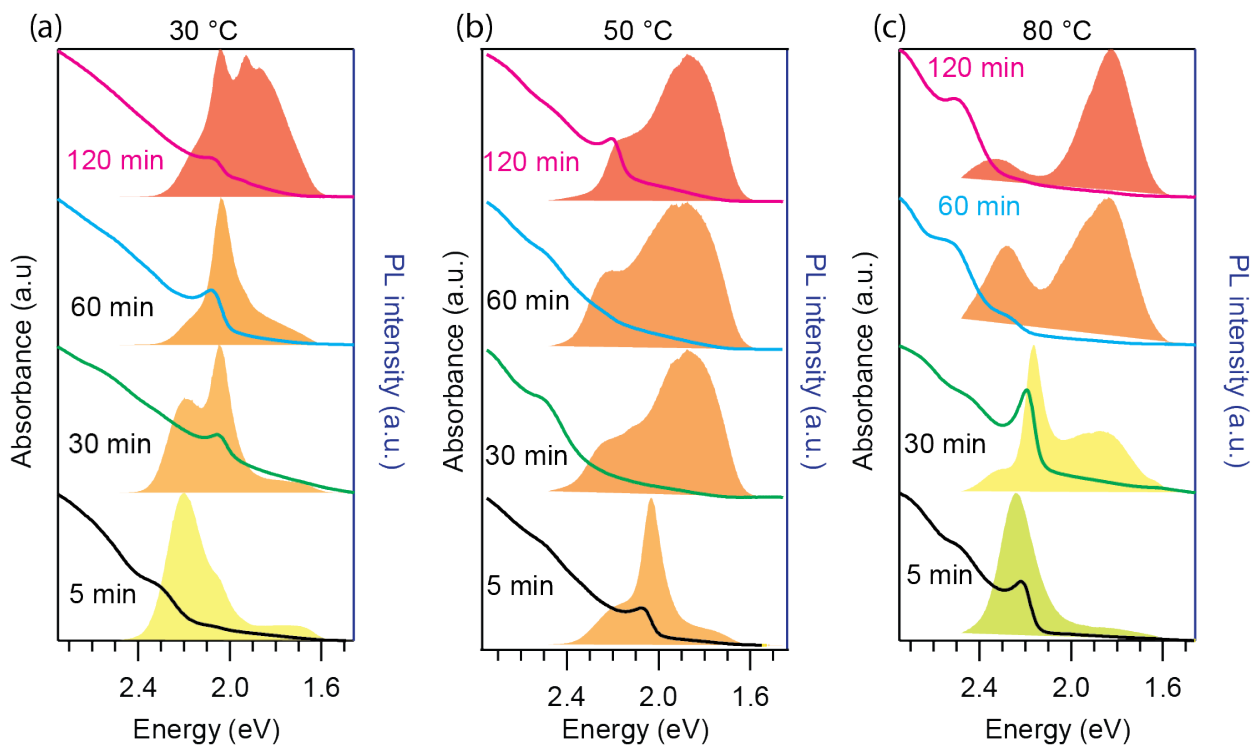

Figure S4: UV-visible absorption (solid line) and photoluminescence spectra (shaded region) of the supernatants collected at different sonication times for syntheses at (a) $30{ }^{\circ} \mathrm{C}$, (b) 50 ${ }^{\circ} \mathrm{C}$, and (c) $80^{\circ} \mathrm{C}$. The left (black) and right (blue) axes represents the absorbance and PL intensity, respectively.

(a)

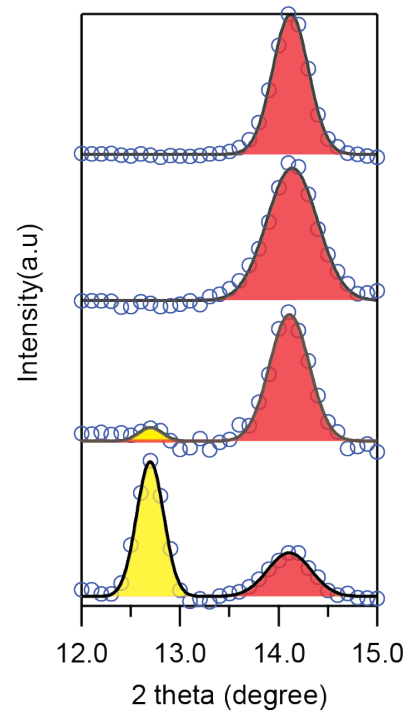

(b)

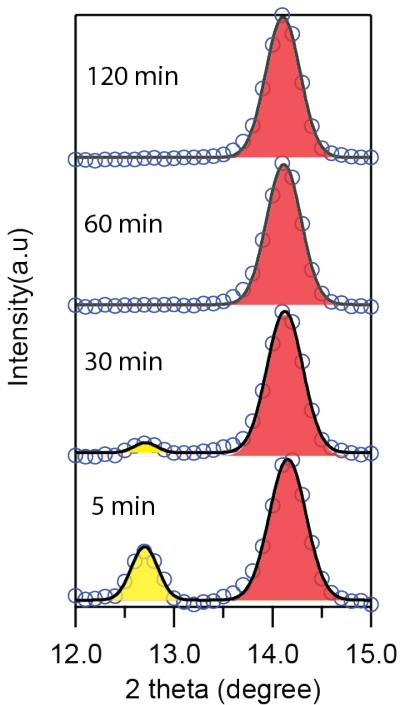

(c)

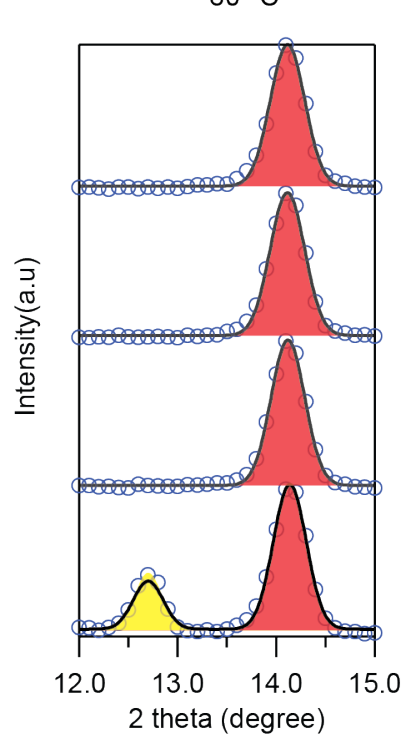

Figure S5: Deconvoluted XRD patterns of the precipitate for 5, 30, 60, and 120 minutes of sonication synthesized at (a) $30^{\circ} \mathrm{C}$, (b) $50^{\circ} \mathrm{C}$, and (c) $80^{\circ} \mathrm{C}$. Here, the blue circle trace represents the raw XRD data, and the black trace represents the fitted XRD data. Here, we have done the Gaussian fitting by Igor pro software. The yellow color represents the amount of lead iodide, and the red color represents the amount of 3D MAPI in cubic phase. 

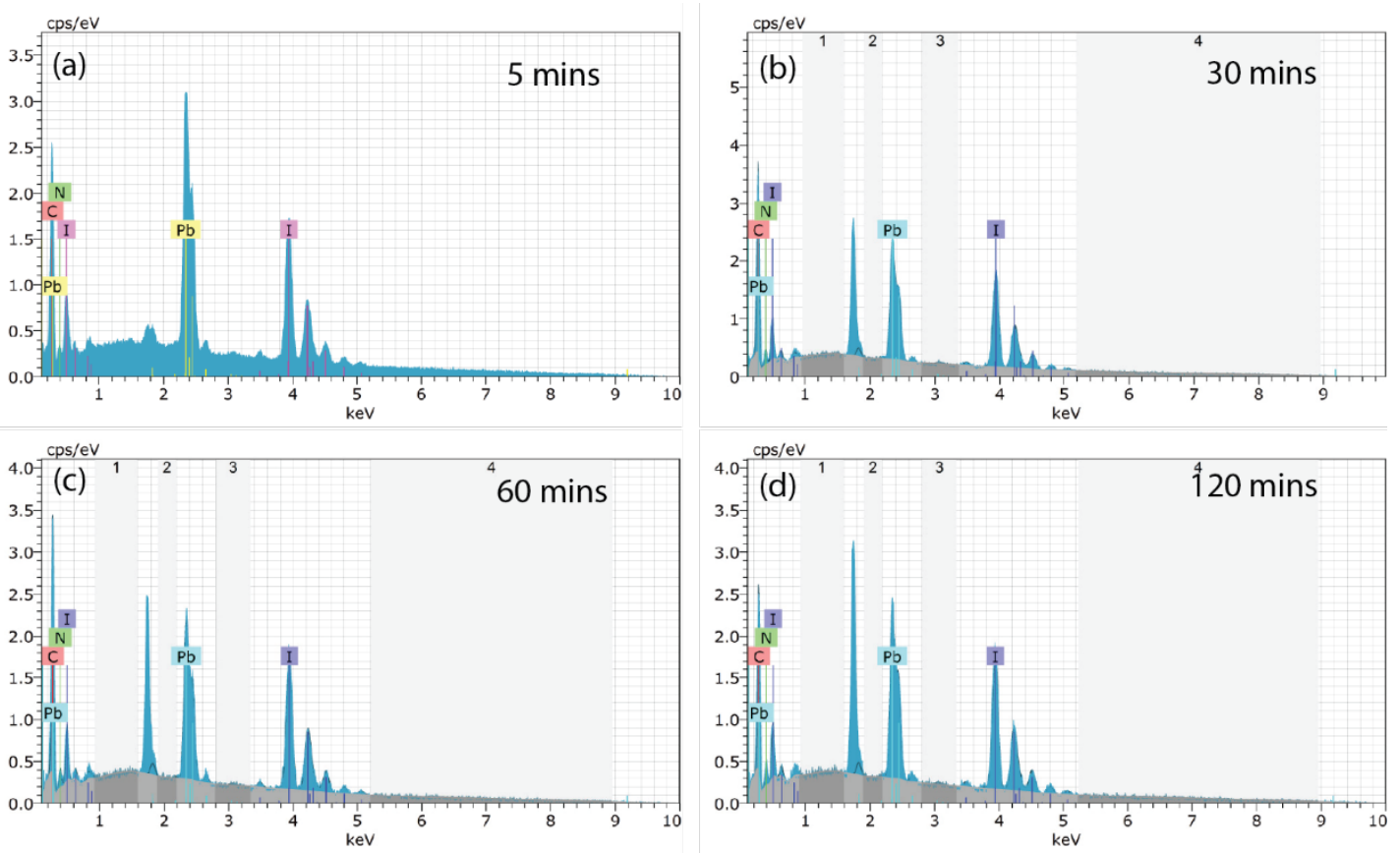

Figure S6: EDX spectra of the precipitates synthesized at $50{ }^{\circ} \mathrm{C}$ for (a) 5 minutes, (b) 30 minutes, (c) 60 minutes and (d) 120 minutes of sonication.

Table S3:

FESEM/EDX elemental atomic concentration (at. \%) of the precipitates synthesized at $50{ }^{\circ} \mathrm{C}$ for 5 minutes, 30 minutes, 60 minutes, and 120 minutes of sonication.

\begin{tabular}{lcccc}
\hline Element & \multicolumn{4}{c}{ Atomic concentration (at. \%) } \\
\hline & $\mathbf{5}$ mins & $\mathbf{3 0}$ mins & $\mathbf{6 0}$ mins & $\mathbf{1 2 0}$ mins \\
\hline Lead $(\mathrm{Pb})$ & $15.6 \pm 4.4$ & $10.3 \pm 3.5$ & $10.5 \pm 3.5$ & $12.4 \pm 3.6$ \\
& & & & \\
lodine $(\mathrm{I})$ & $34.1 \pm 5.5$ & $30.2 \pm 5.9$ & $31.6 \pm 6.0$ & $36.9 \pm 6.2$ \\
& & & & \\
\hline
\end{tabular}




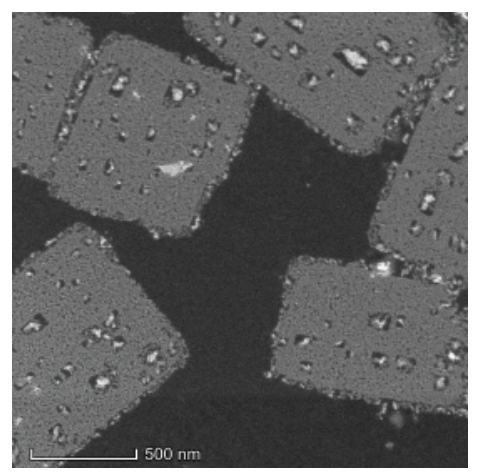

Figure S7: Dark filed image of bigger nanoplatelets collected from the 5 minutes of the reaction precipitate.

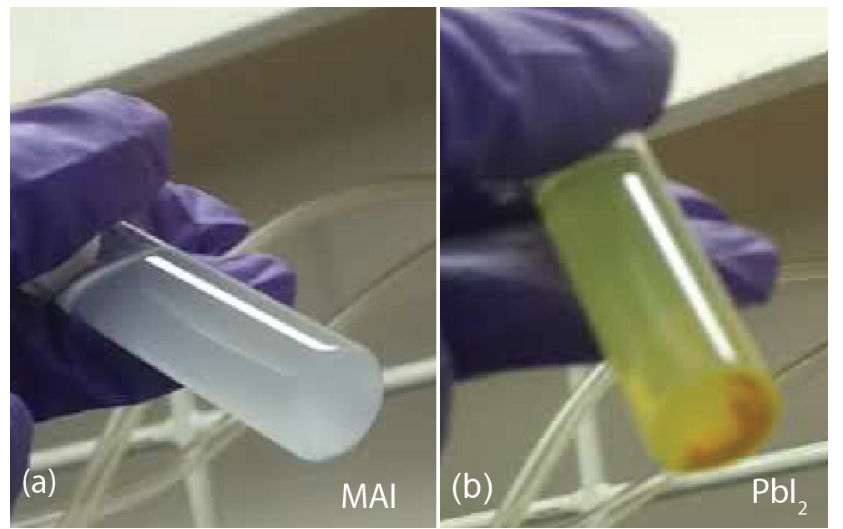

Figure S8: (a) MAI, and (b) $\mathrm{Pbl}_{2}$ in toluene with oleylamine as a ligand after sonication for 20 minutes at $30^{\circ} \mathrm{C}$.

\section{Reference}

(1) Liu, J.; Leng, J.; Wu, K.; Zhang, J.; Jin, S. Observation of Internal Photoinduced Electron and Hole Separation in Hybrid Two-Dimentional Perovskite Films, J. Am. Chem. Soc. 2017, 139, 1432 - 1435. 